\title{
Relationship Between Strain and Electrical Resistivity of Vulcanized Rub- ber Filled with Carbon Nanofiber
}

\author{
L. Bao ${ }^{* 1}$, K. Todaka ${ }^{1}$, I. Yamaura ${ }^{1}$, Z. Pan ${ }^{2}$, N. Amino ${ }^{3}$ and K. Kemmochi ${ }^{1}$ \\ ${ }^{1}$ Faculty of Textile Science and Technology, Shinshu University, 3-15-1 Tokida, Ueda, 386-8567, Japan \\ ${ }^{2}$ School of Material Engineering, Soochow University, Suzhou, Jiangsu 215021, China \\ ${ }^{3}$ Yokohama Rubber Company, Ltd., 1-7-7 Shinomiya, Hiratsuka City, 254-8601, Japan
}

\begin{abstract}
Rubber material generally behaves as an electrical insulator, but vapor-grown carbon fiber (VGCF) is known for its electrical conductivity. Thus, we compounded styrene-butadiene rubber and carbon nanofiber (VGCF), and studied the relationship between strain and the DC electrical resistivity of vulcanized rubber filled with carbon nanofiber.

No change of hardness was observed in hardness tests. VGCF has a reinforcement effect similar to carbon black (CB). The volume electrical resistance decreases with an increase in VGCF concentration, and the relationship is nonlinear. The volume electrical resistance of rubber with VGCF increased when the sample was extended. The change rate is related to the parts and contents of the fillers. The relationship between strain and electrical resistance of rubber with VGCF is suitable for application as a strain sensor.
\end{abstract}

Keywords: Electrical volume resistivity, carbon nanofiber, rubber, strain, hardness, measurement, electrodes.

\section{INTRODUCTION}

Carbon nanofiber (CNF) is known for its heat conductivity, electrical conductivity, and strength. It is used for electrodes, improving electro-conductivity in glue and metal, and increasing material strength, based on these qualities. In addition, a previous study reported that the characteristics of carbon nanotubes were equal to those of carbon nanofiber (CNF) [1].

Vapor-grown carbon fiber (VGCF) is a type of carbon nanofiber that differs from other types of carbon nanotubes in its production method, its unique physical characteristics, and the prospect of low-cost fabrication. The structure and properties of VGCF were described in considerable detail in two recent publications [1 and 2].

VGCF tends to have a smaller diameter and a larger aspect ratio than other short carbon fibers. A recent report demonstrated that conventional thermoplastic can be reinforced with VGCF in a straightforward manner via traditional injection moulding [3 and 4]. The reinforcement of rubbery matrices by vapor-grown carbon nanofiber (VGCF) was reported by Gauthier [5]. An evaluation of the mechanical performances revealed a linear increase of the modulus measured above and below the glass transition temperature for the nanofiber content.

Rubber material generally behaves as an electrical insulator. However, it can exhibit electrical responses within a certain range if conductive fillers are added as a second phase. For example, electro conductivity was demonstrated in carbon nanofiber filled with resin [6]. Polypropylene filled with vapor-grown carbon-fiber composites exhibits the characteristic percolating behavior of electrical resistance. Appli-

*Address correspondence to this author at the Faculty of Textile Science and Technology, Shinshu University, 3-15-1 Tokida, Ueda, 386-8567, Japan, E-mail: baolimin@shinshu-u.ac.jp cation of an electro-conductive composite using vaporgrown carbon fiber to EMI shielding material has also been reported [7]. Composites using VGCF are considered to be highly conductive plastic materials with sufficient strength that could be applied to advanced EMI shielding technology.

Rubber generally allows relatively large deformation at breaks. We can apply rubber filled with VGCF to a large deformation strain sensor if the electrical resistance is related to deformation. However, there are no studies that clarify the relationships of the strain of CNF (VGCF) and rubber and the electrical characteristics. Therefore, there are many unknown factors in this area.

We compounded styrene-butadiene rubber and carbon nanofiber (VGCF) in this study. We then investigated the mechanical characteristics of nanofiber and rubber, and measured the electrical characteristics of vulcanized rubber filled with carbon nanofiber to develop a large strain sensor for rubber. The relationships of strain and volume resistivity were also examined.

\section{EXPERIMENT}

\section{Sample Materials}

A vapor-grown carbon fiber (VGCF, Showa Denko KK) was used to fabricate the rubber composites for this study. The VGCF were measured by scanning electron microscopy (SEM); their average diameter was $0.15 \mu \mathrm{m}$ and their average length, $15 \mu \mathrm{m}$. The real density of VGCF is $2 \mathrm{~g} / \mathrm{cm}^{3}$, and the resistivity is $10^{-4} \Omega \mathrm{cm}$. CB (N660 Carbon Black, Tokai Carbon Co., Ltd.) was used as filler for the rubber reinforcement. We used SBR 1502 (styrene-butadiene rubber, styrene content $=23.5 \mathrm{wt} \%$, Zeon Co., Ltd.) for the matrix.

We prepared styrene-butadiene rubber (SBR 1502, Zeon Co. Ltd.) and changed the ratio of carbon black (N660 black, Tokai Carbon Co. Ltd.) and VGCF (Showa Denko KK). The components listed in Table 1, except for sulfur and TBBS, 
were mixed for five minutes using a banbury mixer with 1.71 chamber volume to obtain a master batch. The master batch, sulfur, and TBBS were then mixed by an open roll mill. The mixed-rubber compounds were cured for 30 minutes at 433.15 Kelvin in the form of sheets $2 \mathrm{~mm}$ in diameter. The VGCF loading in the samples varied from 0 to 60 PHR (parts per hundred rubber, weight), as indicated in Table $\mathbf{1}$, to examine the effect of filling singly. We prepared the rubber when the filler parts were constant, and changed the ratio of the CB and VGCF in seven phases to examine the effect of electricity and the mechanics of rubber filled with VGCF. In addition, a sample filled with the same ratio of VGCF and $\mathrm{CB}$ was made to examine the electric characteristics when $\mathrm{VGCF} / \mathrm{CB}$ is constant.

Tables 2, $\mathbf{3}$ and $\mathbf{4}$ present the composition of multiple sample materials. Other fillers, except $\mathrm{CB}$ and VGCF, were the same as in Table 1. Here, Sample Nos. 2-1 and 4-1 are the same as Sample No. 1-1. Sample No. 3-7 is the same as Sample No. 1-7. Sample No. 4-4 is the same as Sample No. 3-4. These samples were prepared by Yokohama Rubber Company, Ltd.

Table 1. Composition of Compounds (Parts per Hundred Rubber)

\begin{tabular}{|c|c|c|c|c|c|c|c|}
\hline Sample No. & $\mathbf{1 - 1}$ & $\mathbf{1 - 2}$ & $\mathbf{1 - 3}$ & $\mathbf{1 - 4}$ & $\mathbf{1 - 5}$ & $\mathbf{1 - 6}$ & $\mathbf{1 - 7}$ \\
\hline \hline SBR 1502 & 100 & 100 & 100 & 100 & 100 & 100 & 100 \\
\hline N660 black & 0 & 0 & 0 & 0 & 0 & 0 & 0 \\
\hline VGCF & 0 & 10 & 20 & 30 & 40 & 50 & 60 \\
\hline ZnO & 3.0 & 3.0 & 3.0 & 3.0 & 3.0 & 3.0 & 3.0 \\
\hline Stearic acid & 1.0 & 1.0 & 1.0 & 1.0 & 1.0 & 1.0 & 1.0 \\
\hline 6PPD & 1.0 & 1.0 & 1.0 & 1.0 & 1.0 & 1.0 & 1.0 \\
\hline Process oil & 5.0 & 5.0 & 5.0 & 5.0 & 5.0 & 5.0 & 5.0 \\
\hline Sulfur & 2.0 & 2.0 & 2.0 & 2.0 & 2.0 & 2.0 & 2.0 \\
\hline TBBS & 1.0 & 1.0 & 1.0 & 1.0 & 1.0 & 1.0 & 1.0 \\
\hline TOTAL & 113.0 & 123.0 & 133.0 & 143.0 & 153.0 & 163.0 & 173.0 \\
\hline
\end{tabular}

Table 2. Composition of Compounds (Parts per Hundred Rubber)

\begin{tabular}{|c|c|c|c|c|c|}
\hline Sample No. & $\mathbf{2 - 1}$ & $\mathbf{2 - 2}$ & $\mathbf{2 - 3}$ & $\mathbf{2 - 4}$ & $\mathbf{2 - 5}$ \\
\hline \hline SBR 1502 & 100 & 100 & 100 & 100 & 100 \\
\hline N660 black & 0 & 50 & 60 & 70 & 80 \\
\hline VGCF & 0 & 0 & 0 & 0 & 0 \\
\hline
\end{tabular}

Table 3. Composition of Compounds (Parts per Hundred Rubber)

\begin{tabular}{|c|c|c|c|c|c|c|c|}
\hline Sample No. & $\mathbf{3 - 1}$ & $\mathbf{3 - 2}$ & $\mathbf{3 - 3}$ & $\mathbf{3 - 4}$ & $\mathbf{3 - 5}$ & $\mathbf{3 - 6}$ & $\mathbf{3 - 7}$ \\
\hline \hline N660 black & 60 & 50 & 40 & 30 & 20 & 10 & 0 \\
\hline VGCF & 0 & 10 & 20 & 30 & 40 & 50 & 60 \\
\hline
\end{tabular}

Table 4. Composition of Compounds (Parts per Hundred Rubber)

\begin{tabular}{|c|c|c|c|c|c|}
\hline Sample No. & $\mathbf{4 - 1}$ & $\mathbf{4 - 2}$ & $\mathbf{4 - 3}$ & $\mathbf{4 - 4}$ & $\mathbf{4 - 5}$ \\
\hline \hline N660 black & 0 & 10 & 20 & 30 & 40 \\
\hline VGCF & 0 & 10 & 20 & 30 & 40 \\
\hline
\end{tabular}

\section{Measurement Apparatus and Method}

A four-point probe was used to eliminate the influence of the ammeter internal resistance when measuring the volume resistance of the rubber filled with VGCF. Fig. (1a) illustrates the electrical circuit for measurement.

The current $(A)$ and the voltage $\left(V_{2}\right)$ of the sample material were measured by two probes and used to calculate the volume resistivity.

Volume resistivity $=R \times \frac{w}{l} \times t=\frac{V_{2}}{I} \times \frac{w}{l} \times t$

where $R$ is the resistance $\left(V_{2} / I\right), I$ is the current, $w$ is the width of the sample material, $l$ is the length, and $t$ is the depth.

The current was measured at room temperature with a Keithley 617 electrometer (Keithley Instruments, Inc.). Measurements of voltage were performed with a Hioki 3801 Digital HiTester (Hioki E.E. Corporation).

Fig. (1b) depicts the tensile apparatus. The sample material was fixed by two clamps, and was extended by moving the clamps.
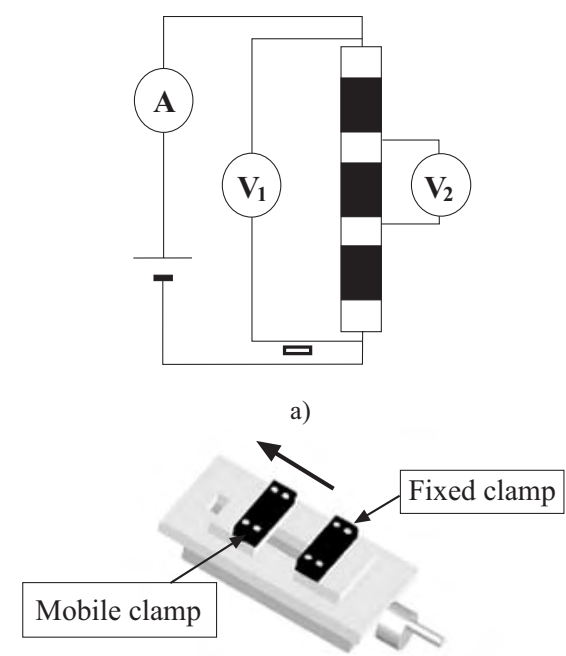

b)

Fig. (1). Experimental device to measure resistance.

a) Electrical circuit for measurement. b)Tensile apparatus.

Each sample had four electrodes; that section of the sample was coated with evaporated platinum. Platinum paste was used to ensure good contact of the sample surface with the electrodes. A copper wire was glued to an electrode of the sample by conductive bonding material (Conductive Epoxy CW2400, Circuitworks Co., Ltd.). The volume resistiv- 
ity of this conductive bonding material was $0.001 \Omega \mathrm{cm}$. The sample material was $36 \mathrm{~mm} \times 5 \mathrm{~mm} \times 2 \mathrm{~mm}$, and the electrodes were $3 \mathrm{~mm}$ wide, with $8 \mathrm{~mm}$ between electrodes. Fig. (2) depicts the sample material. The experiment was repeated five times.

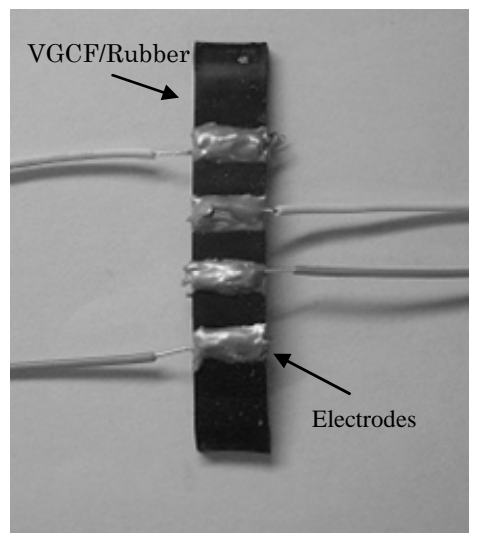

Fig. (2). Sample material.

The hardness of the samples was obtained by the spring type hardness tester (Shimadzu Co., Ltd.; type 200) at room temperature to determine the mechanical characteristics of rubber filled with carbon nanofiber. The geometry of the samples and the experiment conditions followed JIS K 6301[9] and ISO48:1994[10].

\section{RESULTS AND DISCUSSION}

\section{Hardness and VGCF-Filling Rate}

Urabe [11] reported that the addition of carbon black as a filler increases the tensile strength and hardness of rubber. We measured the relationships of hardness and the VGCF filling rate of VGCF-filled rubber when the sum of the filling weights of the $\mathrm{CB}$ and VGCF was constant to examine the mechanical characteristics reinforcement effect of VGCF. Fig. (3) illustrates the relationship between the hardness of rubber and filler rate (weight, PHR) in the VGCF based on the spring type hardness tests results. The sample is No. 3 (Table 3). No change in hardness was found throughout the experimental range for all samples. Thus, VGCF has a hardness effect similar to CB.

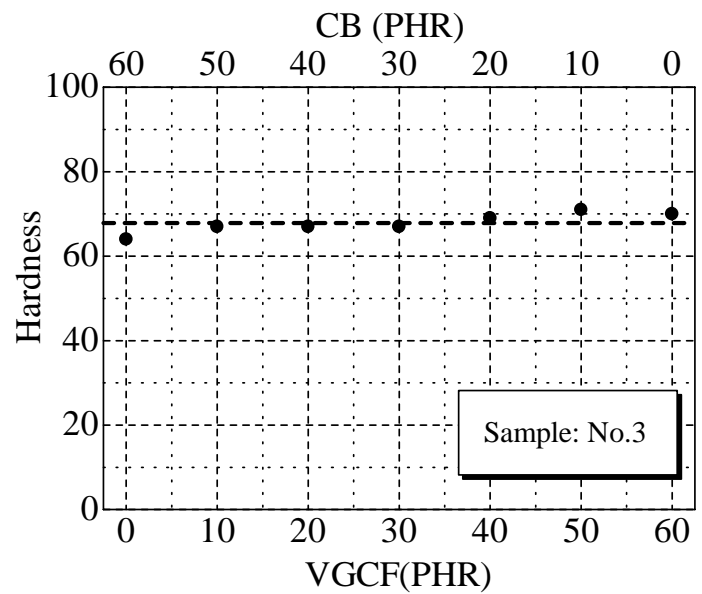

Fig. (3). Relationship between VGCF filling rate (PHR) and hardness by the spring type hardness tester.

\section{Time and Resistivity}

The electric current changes with time when voltage is applied to a sample. The electrical resistance $\left(\mathrm{V}_{2} / \mathrm{I}\right)$ decreases with time. Fig. (4) presents an example of the measurement results. The sample is No. 1-6 (Table 1). The current became steady at $1800 \mathrm{sec}$, as indicated in Fig. (4). Therefore, $1800 \mathrm{sec}$ was selected as the sampling time for measurements in this research.

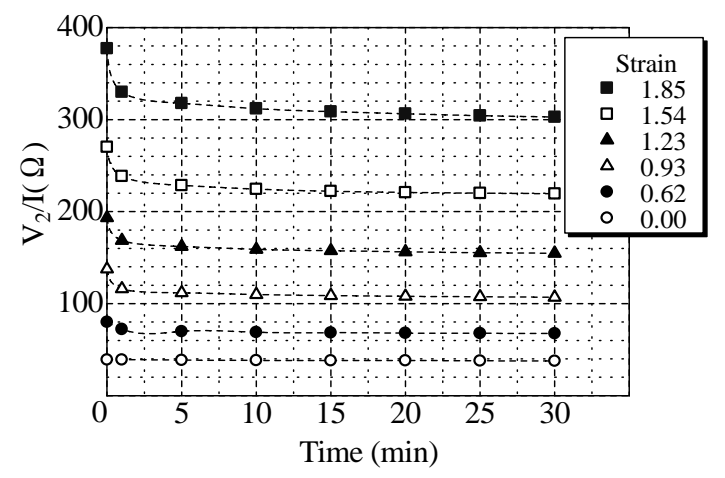

Fig. (4). Relationship between resistance and time. (Sample No. 1$6)$.

\section{Volume Electrical Resistivity of Rubber Filled with VGCF}

The effect of the filler concentration on the volume electrical resistivity of rubber is presented in Fig. (5). "Only CB" designates data of measurements of the samples of Table 2. We could not measure it using the Keithley 617 electrometer under 60PHR, since the current was minimal. "Only VGCF" designates data for the samples in Table $\mathbf{1}$, "VGCF/CB $=$ Con." are data for the samples in Table $\mathbf{4}$, and "VGCF $+\mathrm{CB}=60$ PHR" are data for the samples in Table 3. The missing points are points at which measurement was impossible. The change became smooth beyond 40PHR. The volume electrical resistance of rubber filled with VGCF is slight compared with rubber and $\mathrm{CB}$. The volume electrical resistance depended on the VGCF concentration, since $\bullet, \mathbf{\Delta}$, and $\Delta$ were slightly different and almost repeat. Fig. (5) indicates that the percolation threshold for rubber and VGCF depends on the applied field.

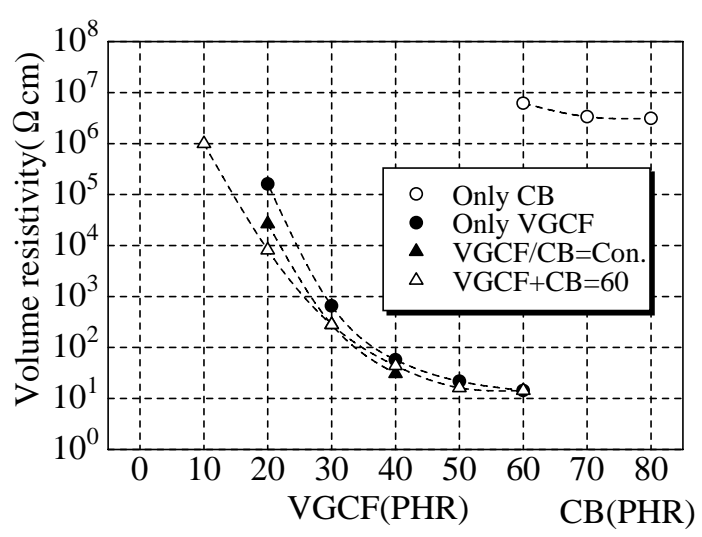

Fig. (5). Volume electrical resistivity of rubber filled with VGCF and $\mathrm{CB}$. 


\section{Volume Resistivity and Strain of the Samples (Only VGCF)}

We measured the electrical characteristics of vulcanized rubber filled with VGCF when the sample was extended as static. Fig. (6) illustrates the relationship between the strain and volume resistivity of sample No.1-. The missing points are points at which measurement was impossible using our apparatus. The volume resistivity and the strain of the sample increased when the sample was extended. The electrode broke away when the stress was over 1.7 and we could not measure the electrical resistance. The electrical resistance of the sample of rubber filled with CB (only CB) was too high when it was extended, and we could not measure it.

The volume electrical resistance change rate $\left(\Delta \rho_{V} / \rho_{V 0}\right)$ in one strain was

$\Delta \rho_{V} / \rho_{V 0}=\frac{\rho_{V}-\rho_{V 0}}{\rho_{V 0}}$

where $\rho_{V 0}$ is the volume electrical resistance when the strain of the sample was zero and $\rho_{V}$ is the volume electrical resistance at one strain of the sample.

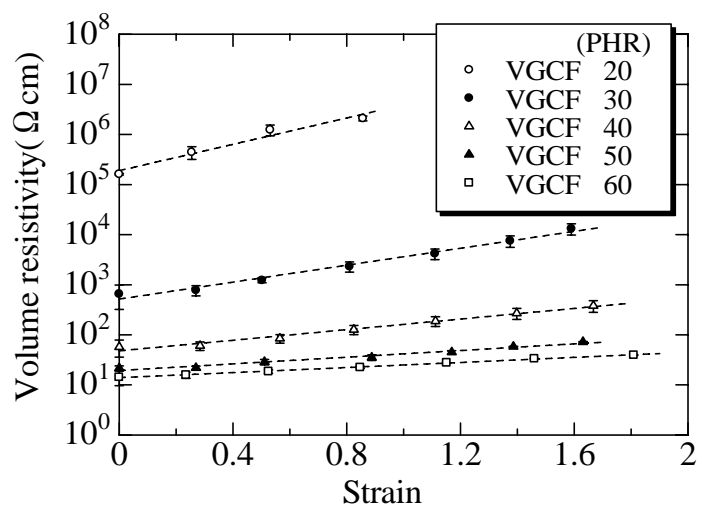

Fig. (6). Relationship between strain and electrical volume resistivity. (Changes in VGCF, CB:0).

Fig. (7) illustrates the relationship between the strain and volume electrical resistance change rate of sample No. 1.

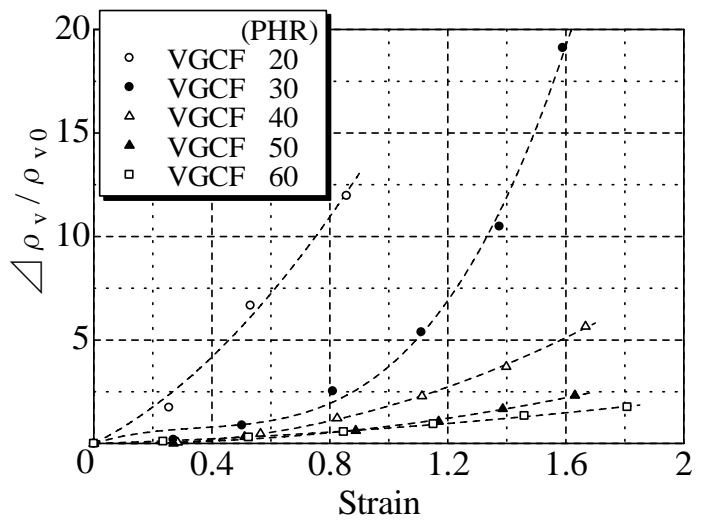

Fig. (7). Relationship between strain and electrical volume resistivity change rate. (Changes in VGCF, CB:0).
The volume resistance change rate increased with decreases in the VGCF concentration, as illustrated in Fig. (7). Fig. (8) presents laser-microscope photographs (OLS3000MS, Olympus Co., Ltd) of the rubber filled with VGCF. Sample No. 1-7. (a) is unstrained, (b) is strained. VGCF is much more rigid than rubber. The contacting fiber separates and the intervals between fibers become large when the sample is extended, as indicated in Fig. (8). Therefore, the volume electrical resistance of the rubber filled with VGCF increases. We can use this relation to measure a large distortion of the rubber.

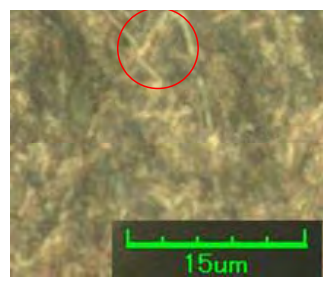

(a)

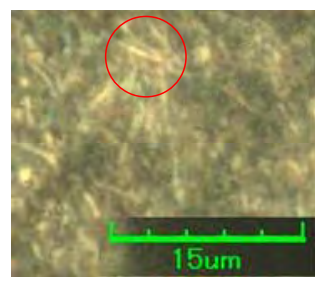

(b)
Fig. (8). Laser microscope photographs of rubber filled with VGCF. (a) is unstrained. (b) is strained.

\section{Volume Resistivity and Strain of Samples $(\mathrm{VGCF}+\mathrm{CB}=$ 60 (PHR))}

Fig. (9) illustrates the relationship between the strain and volume resistivity of sample No.3. The filler parts remained constant (60 PHR). The volume resistivity and the strain of the sample increased, as indicated in Fig. (9). Fig. (10) illustrates the relationship between strain and the volume electrical resistance change rate of a sample. The change of volume resistivity of sample No. 3-3 $(\mathrm{VGCF}+\mathrm{CB}=20+40)$ was remarkable, and it is suitable for application as a strain sensor.

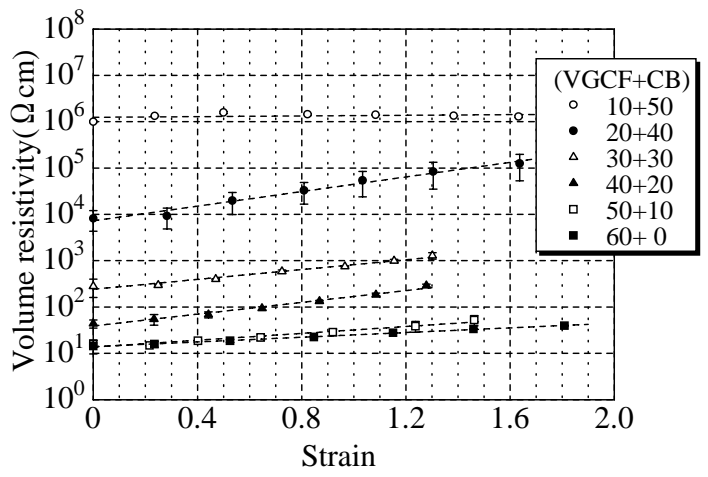

Fig. (9). Relationship between strain and electrical volume resistivity. (VGCF+CB=60 PHR).

\section{Electrical Volume Resistivity and Strain of Samples (VGCF: $\mathrm{CB}=$ Constant)}

Fig. (11) and (12) illustrate the relationship between strain and electrical volume resistivity and the electrical resistance change rate of sample No. 4. The VGCF/CB is constant. The change in electrical volume resistivity of sample No. 4-3 (VGCF/CB=20/20) is remarkable.

\section{CONCLUSIONS}

We prepared styrene-butadiene rubber and VGCF to investigate its mechanical and electrical characteristics. 


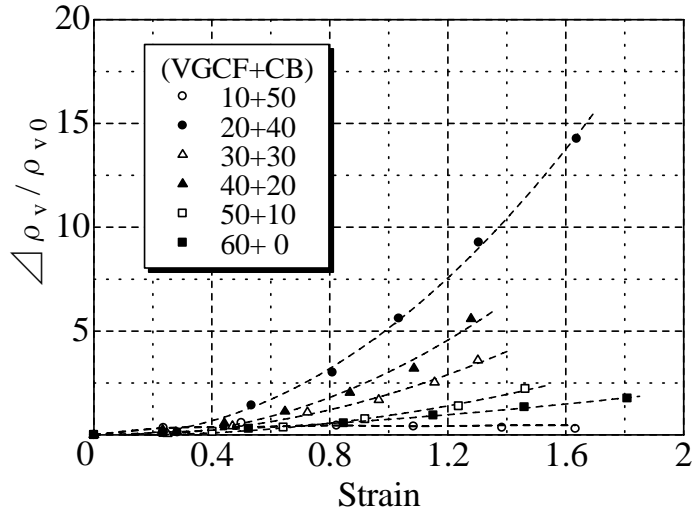

Fig. (10). Relationship between strain and electrical volume resistivity change rate. $(\mathrm{VGCF}+\mathrm{CB}=60 \mathrm{PHR})$.

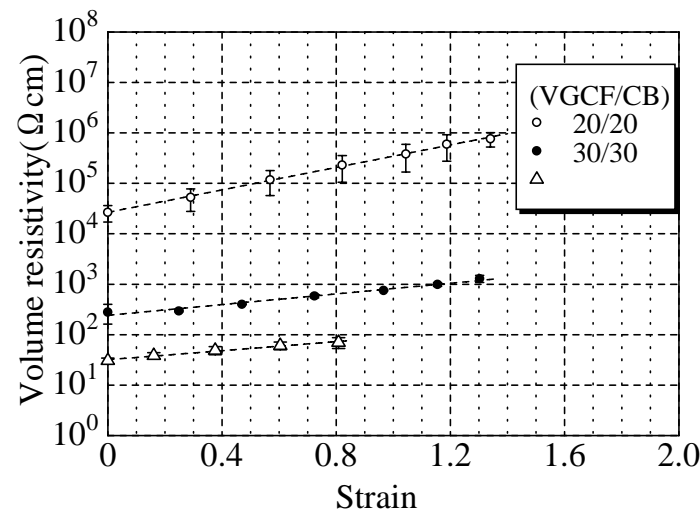

Fig. (11). Relationship between strain and electrical volume resistivity. (VGCF/CB=constant).

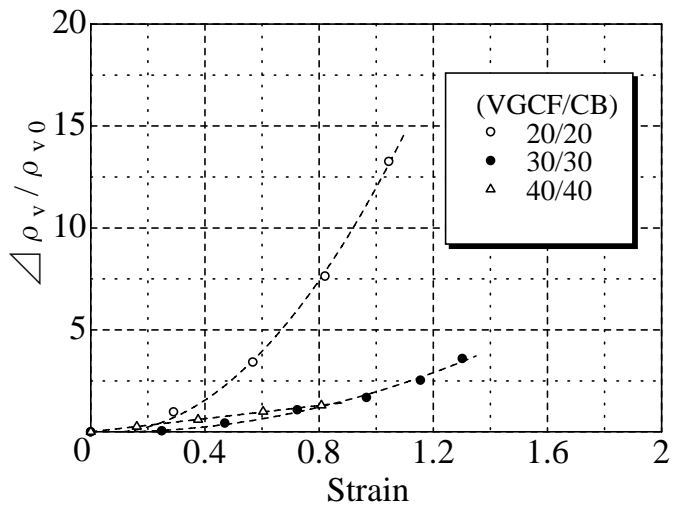

Fig. (12). Relationship between strain and electrical volume resistivity change rate. $(\mathrm{VGCF} / \mathrm{CB}=$ constant $)$.
No change in hardness was recorded in hardness tests. VGCF has a hardness effect similar to that of CB.

The volume electrical resistance decreases with an increase in VGCF concentration, and the relationship is nonlinear. The volume electrical resistance of rubber and VGCF increased when the sample was extended; the change rate was related to the parts and contents of the fillers. The relationship between the strain and electrical resistance of rubber and VGCF is suitable for application as a strain sensor.

\section{ACKNOWLEDGMENTS}

This research was supported by CLUSTER of the Ministry of Education, Culture, Sports, Science and Technology, Japan.

\section{REFERENCES}

[1] Shinohara H. Recent Advances in the Research and Development of Nanocarbon Materials, CMC Publishing Co. Ltd, 291-299, 2003.

[2] Tibbetts GG, Doll GL, Gorkiewicz DW, et al. Carbon 1993; 31: 1039-1047.

[3] Carneiro OS, Covas JA, Bernardo CA, et al. Compos Sci Technol 1998; 58: 401-407.

[4] Hattum FWJ, Bernardo CA, Finegan JC, Tibbetts GG, Alig RL, Lake ML. Polym Compos 1999; 20: 683-688.

[5] Gauthier C, Chazeau L, Prasse T, Cavaille JY. Composites Sci Technol 2005; 65(2): 335-343.

[6] Gordeyev SA, Macedo FJ, Ferreira JA, Bernardo CA, physica B 2000; 279: 33-36.

[7] katsumata M, Yamanashi H, Ushijima H, Endo M, T. IEE Japan 1993; 113-A(6): 473-479.

[8] Matsuzaki R, Todoroki A, Kobayashi H, Shimamura Y, J. Japn Soc Composite Mate 2004; 30(2): 55-62.

[9] Japanese industrial standard, JIS K 6301 Rubber, vulcanized or thermoplastic -- Determination of tensile stress-strain properties, 2004.

[10] ISO 48: 1994, Rubber, Vulcanized or thermoplastic -Determination of hardness.

[11] Urabe T, Property and processing of rubber, vol.7, 268-271. Chijin Shokn, 1965. 